\title{
Formation d'ingénieur en Hydraulique et Mécanique des fluides : exemple de l'enseignement à l'Ecole nationale des Ponts et Chaussées
}

\author{
P.L. Viollet** et J. P. Benque* \\ Electricité de France, Direction des Etudes et Recherches. \\ (*) Professeur à l'ENPC. \\ (**) Professeur-Adjoint à l'ENPC.
}

\section{Introduction}

L'Ecole nationale des Ponts et Chaussées est sans doute, parmi les Grandes Ecoles françaises, celle dont le profil est traditionnellement le plus orienté dans la direction de la Mécanique. Aujourd'hui, ses filières techniques, Filière Génie civil, Génie industriel, Informatique et Mathématiques appliquées, reflètent la diversité des débouchés de ses ingénieurs, dont la formation initiale repose pour beaucoup sur les Sciences mécaniques et leurs applications.

De nos jours, la discontinuité entre Hydraulique et Mécanique des fluides, qui était encore relativement forte voici une dizaine d'années, s'estompe sous l'effet d'une communauté de préoccupations et de moyens d'étude. Ce dénominateur commun est la modélisation, processus qui consiste à formuler, via un jeu d'hypothèses, une description simplifiée de la réalité, puis à bâtir un modèle, modèle physique (souvent appelé aussi modèle réduit) ou modèle reposant sur une résolution numérique d'un jeu d'équations différentielles. L'un comme l'autre conduisent à un travail de réflexion sur la physique : en premier lieu sur les équations de la mécanique des fluides et souvent de la thermodynamique, voire même de la chimie. Ce travail de réflexion permet, pour chaque classe de cas particuliers de formuler les hypothèses de modélisation les plus judicieuses.

Un enseignement général en Mécanique des fluides contient donc nécessairement de multiples composantes qui s'épaulent et s'enrichissent mutuellement. Les auteurs tentent ici, à travers l'analyse de leur action et de celle de leurs collègues de l'équipe enseignante à l'ENPC, d'analyser les synergies multiples entre toutes ces composantes.
2. Organisation de l'enseignement de Mécanique des fluides à l'ENPC

Cet enseignement intervient en deuxième et troisième années d'étude, et fait suite aux enseignements de base de Mécanique des milieux continus dans lesquels les équations de Navier Stokes et leurs applications aux fluides parfaits (non visqueux) et au modèle d'écoulement irrotationnel ont déjà été introduits.

Par ailleurs, en amont des quatre modules dont la description suit, un module sur les méthodes de résolution, commun avec nos collègues de Mécanique du solide a été mis en place : ce module contient, en une quinzaine d'heures, un exposé assez détaillé sur la méthode des caractéristiques, ce qui permet d'utiliser plus facilement par la suite ces notions pour l'étude des problèmes de propagation.

Un certain nombre de notions de base étant ainsi posées, l'enseignement est donc dès le début en mesure de s'orienter vers des modèles applicables à certains problèmes réels d'écoulements. L'enseignement est découpé en modules de chacun 35 heures environ.

\section{a) Module de Mécanique des fluides appliquée}

C'est le seul module qui soit obligatoire pour l'ensemble des élèves de l'ENPC, toutes fillières confondues. Le fil conducteur de ce module est la modélisation. Il est divisé en gros en deux parties. La première met en lumière le lien entre le choix des grandeurs que l'on souhaite connaitre et la "loi de comportement» du "matériau» (turbulence).

The present paper deals with the complement aspects of engineering trainings in both Hydraulics and Fluid Mechanics, which results from the increasing use of the general Fluid Mechanics methods while solving Hydraulics problems. The paper emphasizes how these complementary aspects are used in the various subtopics of the Applied Fluid Mechanics course at "Ecole Nationale des Ponts et Chaussées ". 
La seconde partie illustre la méthodologie pour mettre en œuvre un modèle géométriquement plus simple.

La première de ces deux parties a donc un caractère extrêmement général. On rappelle tout d'abord les équations générales de la Mécanique des fluides, puis on introduit des outils qui sont des outils de base de l'ingénieur hydraulicien :

- l'analyse dimensionnelle, qui sous-tend toutes les études de dimensionnement de modèles réduits en hydraulique ou en aérodynamique, et qui, au-delà de cette modélisation expérimentale, est une démarche intellectuelle d'analyse commune à toutes les branches de la Mécanique des fluides ;

- les notions de pertes de charge, outils de base des dimensionnements des circuits hydrauliques.

Après ces notions qui ont permis de mettre un pied dans la résolution de nombreux problèmes concrets, est abordée une introduction à la turbulence, sujet difficile s'il en est. L'introduction qui est faite s'appuie sur les bilans énergétiques (équation d'énergie cinétique turbulente, en particulier), et introduit de façon un peu heuristique le modèle de Kolmogorov pour l'analyse des échanges énergétiques aux différentes échelles de l'écoulement turbulent. Les méthodes de l'ingénieur (modèle « $k$-epsilon » notamment) sont introduites et illustrées. L'écoulement turbulent entre deux parois parallèles est un cas « simple » qui illustre cette modélisation et permet de mener les calculs à terme tout en introduisant les concepts fondamentaux de la description des couches limites. Un autre type d'écoulement de très grande portée générale est abordé ensuite : les écoulements autour d'obstacles et les efforts qui en résultent ; à partir du modèle irrotationnel qui permet d'introduire le concept de masse ajoutée, et celui de portance, la description expérimentale des phénomènes de trainée et de sillage est présentée.

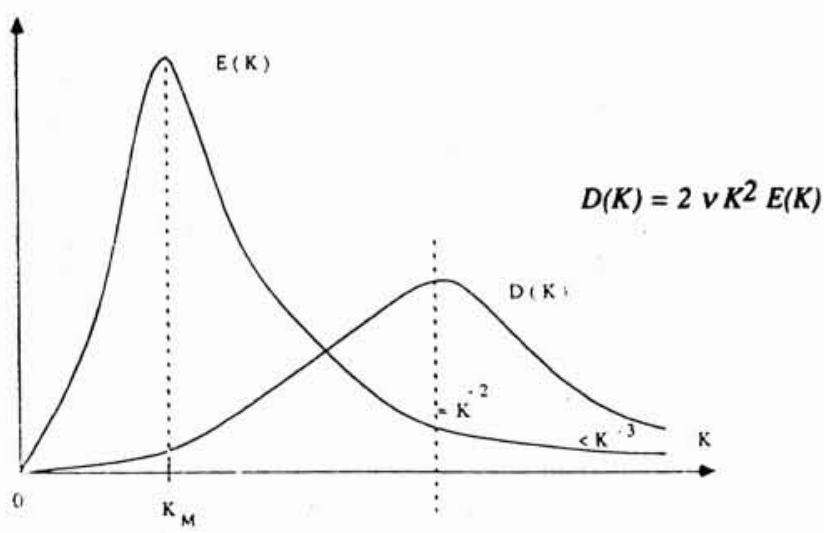

1. Spectres d'énergie turbulente $E(K)$ et de puissance dissipée $D(K)$ en fonction de la norme du vecteur d'onde $K$ (échelles linéaires).
La deuxième partie de ce module repose sur le modèle d'écoulement unidimensionnel, qui permet des résolutions analytiques et une introduction pédagogique des phénomènes de propagation. Ces derniers sont introduits grâce à la théorie des caractéristiques, qui est mise en œuvre pour traiter, en négligeant le frottement, le problème du coup de bélier en conduite, la propagation des ondes de gravité dans le cadre des équations de Saint-Venant pour les ondes longues: application aux ondes de ruptures de barrage, aux régimes transitoires en canal. La notion de choc, de ressaut hydraulique, apparaît comme une conséquence nécessaire des phénomènes de propagation (ondes de compression).

Introduisant ensuite le frottement (coefficients de Chezy et de Strickler), mais en se limitant aux régimes permanents, l'enseignant traite ensuite les lignes d'eau qui permettent de rationaliser et d'expliquer les formes d'écoulements à surface libre que chacun peut observer dans la vie de tous les jours (déversements, passage sur seuil, ...).
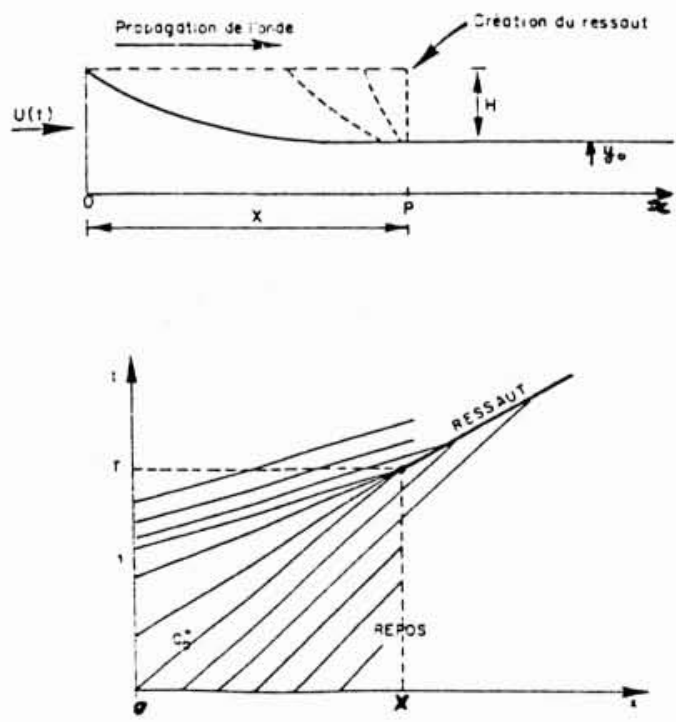

2. Formation d'un ressaut hydraulique en canal (exercice d'application de la méthode des caractéristiques).

Un canal hydraulique de section rectangulaire est rempli d'eau au repos (tirant d'eau y0). A l'origine de ce canal (point $\mathrm{O}$ ), on dispose d'une pompe à débit variable et on désire fabriquer dans le canal, à partir d'un point $\mathrm{P}$ situé à une distance $\mathrm{X}$ de l'origine $\mathrm{O}$, un ressaut hydraulique de hauteur $\mathrm{H}$ 


\section{b) Module d'aérodynamique et transferts de chaleur}

Ce module n'est obligatoire que pour les élèves de la filière Génie industriel de l'ENPC, filière pour laquelle une connaissance des phénomènes de transfert de chaleur est jugée indispensable. Il a cependant une portée très générale, comme le prouvent les exemples illustrant le cours, qui sont empruntés à la fois à la Mécanique des fluides industrielle et aux écoulements dans l'environnement.

L'unité de ce module est l'étude des écoulements à température et/ou masse volumique variable. Après une revue des équations générales de l'aérodynamique, ce module traite des écoulements unidimensionnels en tuyère, qui sont le pendant des écoulements unidimensionnels en canal étudiés dans le premier module. Le parallèle entre les écoulements compressibles et les écoulements à surface libre est ainsi largement développé, les transitions supersonique/choc/subsonique renvoyant aux transitions torrentiel/ressaut/fluvial. L'acoustique est effleurée au passage.

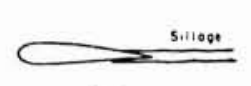

10:

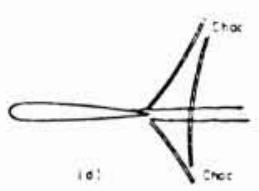

3. Ondes de choc autour d'une aile d'avion, pour des valeurs croissantes du nombre de Mach (de (a) a (e), $M=0,75 \dot{a} 1,4)$.

La deuxième partie de ce module concerne les écoulements soumis aux forces de gravité résultant de différences de température ou de densité, mais dans le cadre simplificateur des petits nombres de Mach: c'est la thermohydraulique. On redécouvre alors la turbulence, avec l'effet de ces forces de gravité sur le mélange turbulent, effet inhibiteur du mélange en stratification stable, ou accélérateur du mélange en stratification instable. Les écoulements stratifiés, qui présentent de nombreuses applications dans l'environnement (inversions de température dans l'atmosphère, thermoclines des lacs ou mers sans marée...) ou dans l'industrie, sont étudiés, en les examinant notamment sous leur modélisation en bi-couche : phénomènes de propagation à l'interface, ondes internes, ressaut hydraulique interne, courants de densité. Revenant à l'écoulement d'un fluide le long d'une paroi, les lois semi-empiriques de coefficient d'échange sont introduites: outils simples du thermicien, elles permettent de calculer l'échange de chaleur dans les problèmes de chauffage ou d'isolation thermique.

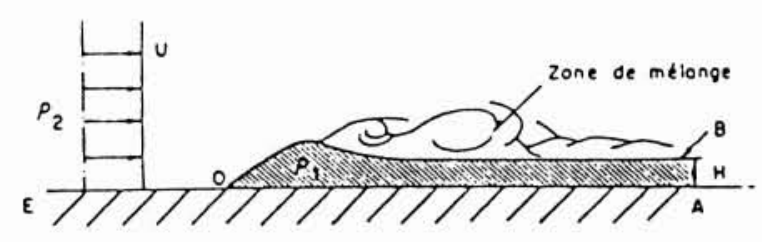

4. Mouvement d'un courant de densité (coin salé en estuaire, front atmosphérique...).

La troisième partie de ce module consiste en une introduction aux méthodes numériques pour résoudre les équations de la mécanique des fluides. Les différentes discrétisations possibles sont introduites, les problèmes de stabilité et de précision des méthodes sont discutés (on s'attache particulièrement à l'étude des équations de transport qui constituent l'un des points les plus sensibles des méthodes numériques en Mécanique des Fluides).

\section{c) Aérodynamique de la combustion}

Ce cours a été plus particulièrement conçu pour les élèves de la filière Génie industriel de l'ENPC. Il est également ouvert aux élèves du DEA de Mécanique de Paris VI.

Prenant pour prétexte l'analyse des différents phénomènes présents dans les brûleurs, chambres de combustion, et systèmes de propulsion, un certain nombre de concepts très généraux sont introduits, dont le champ d'application dépasse en fait celui de la combustion.

Une analyse est tout d'abord faite de la modélisation des écoulements diphasiques. On introduit l'analyse Lagrangienne, puis l'analyse Eulérienne, et le problème de la modélisation des écoulements dispersés turbulents est plus particulièrement discuté (écoulements à bulles, à gouttes, à particules). Les applications de ces méthodes d'analyse sont en fait très larges, bullages en bain liquide, transport de suspensions, en plus des applications spécifiques à la combustion.

L'enseignant aborde ensuite la mise en équations générales des écoulements réactifs en simple phase. Les plasmas et les arcs électriques sont décrits à titre d'illustration, mais c'est dans le domaine de la combustion que se poursuit alors l'enseignement. Combustion en milieu homogène (ignition spontanée, limites d'inflammabilité), puis ondes planes de combustion (détonations, déflagrations), flammes laminaires et flammes turbulentes.

La synthèse entre les formulations étudiées pour les écoulements diphasiques et pour les écoulements réactifs en simple phase est réalisée par l'analyse de la combustion de charbon pulvérisé.

Ce cours se rapporte à des domaines dans lesquels l'évolution des recherches et des connaissances est très rapide, et s'efforce de suivre ces évolutions en donnant au mieux possible un état de l'art de la modélisation dans ces domaines. 

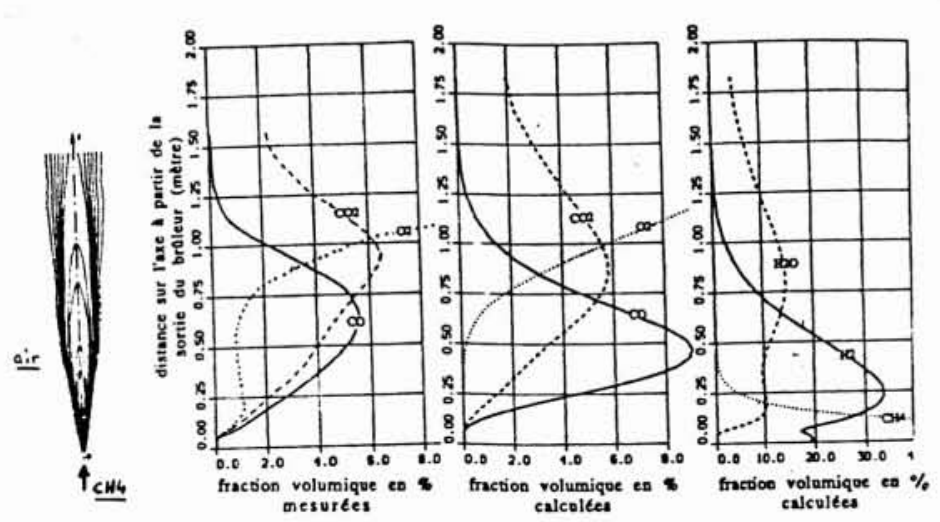

5. Champs de température et de concentrations en espèces chimiques mesurés et calculés dans une flamme de diffusion turbulente.
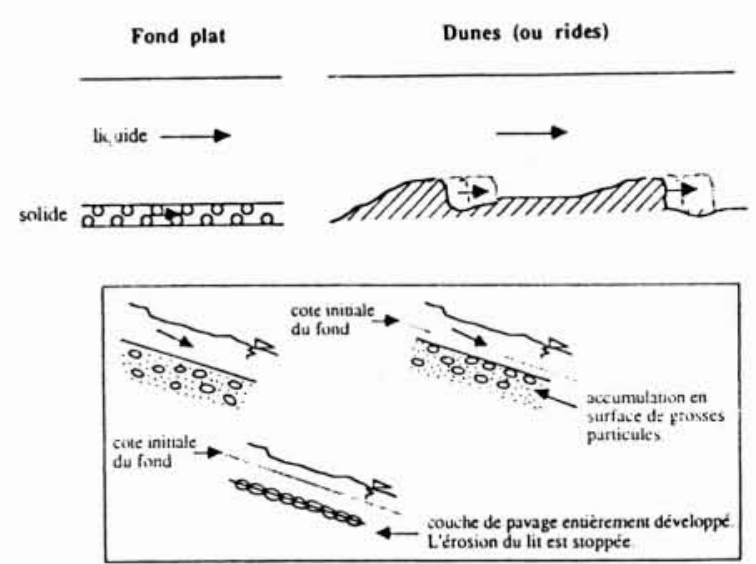

6. En haut : transport de sédiments par charriage en rivière, évolution des dunes. En bas: tri granulométrique et pavage.

\section{d) Grands ouvrages hydrauliques}

Ce module s'adresse plus particulièrement aux élèves de la filière Génie civil. En effet, les cours d'eau naturels sont le résultat d'un équilibre très fin entre les processus de dépôt et d'érosion de sédiments. Un aménagement mal étudié sur une rivière peut provoquer une rupture de cet équilibre et avoir des conséquences considérables sur l'évolution ultérieure du lit naturel.

Dans une première partie, on aborde la description du transport solide en rivière, qui est un des phénomènes essentiels pour la prédiction de l'impact des aménagements sur l'évolution des cours d'eau : analyse dimensionnelle des écoulements liquides transportant des sédiments, transport solide par charriage, ondes de sable (dunes), stabilité des canaux et des lits naturels, suspensions.

Une deuxième partie concerne la modélisation couplée de l'écoulement et du transport solide. L'enseignant reprend tout d'abord en les enrichissant les notions introduites dans le premier module sur les équations de Saint-Venant, et les phénomènes de propagation en rivière. Les exemples importants des crues et des ondes de rupture de barrages sont particulièrement développés, avec un éclairage particulier sur la modélisation numérique de ces phénomènes de propagation. Pour le charriage, ensuite, la description physique de la complexité des phénomènes observés (dunes par exemple, ou encore nécessaire prise en compte de granulométries étendues) appuie la description et la formulation des modèles simplifiés qui sont aujourd'hui utilisables. Les suspensions sont ensuite étudiées.

L'enseignement s'intéresse également à l'aspect non visible des écoulements naturels : celui des nappes souterraines. Après une introduction descriptive, on introduit la loi dynamique de Darcy, puis l'on s'intéresse aux phénomènes de diffusion et dispersion de solutés transportés en milieu poreux (application aux études de pollution des nappes souterraines).
Une dernière partie concerne les ouvrages eux-mêmes : prises d'eau, barrages, évacuateurs de crues.

\section{Analyse des synergies entre les différents modules de Mécanique des fluides}

Dans cet enseignement, hydraulique, aérodynamique se retrouvent de façon imbriquée. Clairement, les similitudes déjà évoquées entre les phénomènes de propagation de l'aérodynamique et des écoulements à surface libre méritent d'être soulignées par l'enseignant, en une vue harmonieuse et unitaire des phénomènes de Mécanique des fluides. Un autre exemple réside dans la turbulence, présente dans toutes les applications de la Mécanique des fluides, en génie industriel comme dans l'environnement. Les méthodes numériques, enfin, sont un puissant dénominateur commun entre les différents domaines de la mécanique des fluides.

Il peut être intéressant de regarder deux de ces exemples de thèmes transversaux à toute la mécanique des fluides : les phénomènes propagatifs, d'une part, et la turbulence, d'autre part.

\subsection{Phénomènes de propagation : modèle unidimensionnel}

Les phénomènes de propagation se retrouvent dans les ondes de gravité à surface libre ou à l'interface de deux fluides de densités différentes, ainsi que dans la propagation acoustique en aérodynamique. Le modèle unidimensionnel (ou modèle filaire) est un outil pédagogique précieux pour l'analyse de ces phénomènes. La notion de propagation juxtaposée avec la non-linéarité des équations de la Mécanique des fluides, conduit à la notion de chocs : choc aérodynamique ou ressaut. 


\begin{tabular}{|c|}
\hline Thème : propagation \\
\hline $\begin{array}{l}\text { Module: Mécanique des fluides appliquée: } \\
\text { - Introduction de la méthode des caractéristiques. } \\
\text { - Application au coup de bélier en conduite. } \\
\text { - Ecoulements fluvial }(u<c) \text { et torrentiel }(u>c) \text {. Nombre de Froude }=u / c \text {. } \\
\text { - Choc : ressaut hydraulique (équations de bilan). }\end{array}$ \\
\hline $\begin{array}{l}\text { Module: Aérodynamique et transfert de chaleur: } \\
\text { - Aérodynamique des tuyères. } \\
\text { - Ecoulements subsonique }(u<c) \text { et supersonique }(u>c) \text {. Nombre de Mach }=u / c \text {. } \\
\text { - Choc: choc aérodynamique. } \\
\text { - Ondes de gravité à l'interface de deux fluides (célérité } c \text { ), courants de densité, ressaut interne. Nombre de Froude réduit }=u / c \text {. }\end{array}$ \\
\hline $\begin{array}{l}\text { Module: Aérodynamique de la combustion : } \\
\text { - Onde plane de combustion : détonation et déflagration. }\end{array}$ \\
\hline $\begin{array}{l}\text { Module: Grands ouvrages hydrauliques: } \\
\text { - Crues. } \\
\text { - Ondes de rupture de barrages. }\end{array}$ \\
\hline
\end{tabular}

\begin{tabular}{|c|}
\hline Thème : turbulence \\
\hline $\begin{array}{l}\text { Module: Mécanique des fluides appliquée: } \\
\text { - Introduction au sujet. } \\
\text { - Bilans énergétiques en écoulement incompressible. } \\
\text { - Modèle de Kolmogorov (spectre en équilibre, grandes et petites échelles). } \\
\text { - Ecoulement incompressible entre deux plans parallèles (couche limite). } \\
\text { - Modèle k-epsilon, autres modèles plus complexes. }\end{array}$ \\
\hline $\begin{array}{l}\text { Module: Aérodynamique et transfert de chaleur: } \\
\text { - Influence des forces de gravité sur bilan d'énergie turbulente. } \\
\text { - Turbulence dans les écoulements stratifiés. } \\
\text { - Transfert de chaleur dans la couche-limite turbulente. } \\
\text { - Turbulence dans les métaux liquides. }\end{array}$ \\
\hline $\begin{array}{l}\text { Module : Aérodynamique de la combustion: } \\
\text { - Turbulence en écoulement diphasique; dispersion. } \\
\text { - Flammes et écoulements réactifs turbulents : problématique. } \\
\text { - Structure des flammes turbulentes (épaisseur de flamme/échelles de longueur de turbulence). Modélisation des flammes turbulentes. }\end{array}$ \\
\hline
\end{tabular}

\subsection{Turbulence}

La présentation de la turbulence à des étudiants contient de nombreuses difficultés pédagogiques. Les élèves ingénieurs qui ont une formation mathématique forte en classes préparatoires, subissent une sorte de choc culturel en abordant un sujet dont la mise en équations est par nature incomplète, et le recours à l'expérience incontournable: sauf à exposer en détail les théories de la turbulence homogène dans l'espace spectral, mettant en évidence l'existence de petites échelles comme des conséquences des interactions non linéaires (triadiques), la cascade énergétique du modèle de Kolmogorov doit être exposée en faisant appel à un sens physique qui en réalité ne s'acquiert qu'après un certain contact expérimental du sujet. Le choix effectué consiste à répartir l'information sur la turbulence sur les différents modules de façon un peu homéopathique, espérant engendrer chez l'étudiant suivant plusieurs de ces modules successifs une formation progressive de son sens physique sur ce sujet.

\section{Conclusions}

Ce papier s'est limité à la description de l'organisation et du contenu des cours de Mécanique des fluides à l'Ecole nationale des Ponts et Chaussées. Il va de soi que l'exposé du cours lui-même se doit d'être complété par des illustrations expérimentales (visites au Laboratoire National d'Hydraulique à Chatou), des documents vidéo, des exemples d'applications concrètes.

Le fil conducteur de cet enseignement est la description des différents aspects de la Mécanique des fluides, appliquée à des domaines aussi divers que l'hydraulique, l'aérodynamique, la combustion, et essayant de jouer sur les similitudes de phénomènes ou de modes d'analyse pour enrichir petit à petit la connaissance physique des écou- lements considérés. Cette connaissance physique est nécessaire à l'élaboration et à la discussion des modélisations qui sont à la base des outils applicables aux problèmes concrets. L'exposé de cette démarche de modélisation appliquée aux différents domaines de la Mécanique des fluides est l'un des fils conducteurs forts de cet ensemble d'enseignements.

\section{Remerciements}

Les auteurs tiennent à remercier leurs collègues membres de l'équipe enseignante de Mécanique des fluides à l'ENPC : R. Cointe, P. Esposito, A. Hauguel, G. Labadie, D. Laurence, J.F. Malherbe, G. Nicollet, R. Pochat, O. Simonin. 